\title{
Desenvolvimento da assimetria manual
}

\author{
Paula Cristina Rodrigues 1 \\ Maria Olga Vasconcelos 1 \\ João Manuel Barreiros 2
}

https://doi.org/10.5628/rpcd.10.01.230

\author{
${ }^{1}$ Faculdade de Desporto \\ Universidade do Porto \\ Portugal \\ ${ }^{2}$ Faculdade de Motricidade Humana \\ Universidade Técnica de Lisboa \\ Portugal
}

\section{RESUMO}

A reduzida tendência para a sinistralidade têm sido documentadas em diversas culturas, em diferentes grupos com patologias identificadas, e em diferentes faixas etárias. Pela importância da funcionalidade manual torna-se pertinente compreender o desenvolvimento do comportamento assimétrico, quer ao nível da preferência quer da proficiência e funcionalidade. O presente ensaio consubstancia-se na descrição do desenvolvimento da preferência e proficiência manual relacionadas com a idade e com o género, e sua caracterização em condições patológicas. As evidências provenientes de vários estudos mostram que a assimetria manual é uma característica que, apesar de se estabelecer durante os primeiros anos de vida, continua a desenvolver-se ao longo da idade. O género, o estado neurológico do sujeito e o tipo de sociedade em que este está inserido são factores que parecem ter efeito no desenvolvimento da preferência manual e na consequente assimetria manual. Pode-se assim dizer que esta resulta de factores biológicos, sociais e culturais característicos de um determinado espaço geográfico e num período de tempo particular.

Palavras-chave: preferência manual, proficiência manual, idade, género, condições patológicas

\section{ABSTRACT \\ Manual asymmetry development}

The reduced tendency to be left-handed has been documented in several cultures, in different groups, with identified pathologies, and with age. The importance of manual dexterity brings additional interest to the problem of the development of asymmetric behavior, either in preference and proficiency. This paper will describe the development of hand preference and proficiency related to age and gender, as well as some aspects of pathological conditions. Evidence from several studies show that manual asymmetry is a characteristic that, although it is set during the first years of life, continues to develop with age. Gender, neurological status, and social characteristics are factors that seem to play a role in the development of hand preference and in the subsequent manual asymmetry. Hand preference and lateral dexterity stems from biological, social, and cultural factors, in a geographical context and in a particular time.

Key-words: manual preference and proficiency, age, gender, pathological conditions 


\section{CONSIDERAÇÕES PRELIMINARES}

A escolha de uma mão em detrimento da outra é o reflexo mais evidente da assimetria no comportamento motor humano. As mãos expressam também diferentes funções na realização de tarefas manuais uma mão assegura funções de suporte ou estabilização e a outra assume papel mais activo.

A tendência para a destralidade e, em contraposição, à reduzida tendência para a sinistralidade, na maioria da população, têm sido vastamente estudadas e documentadas em diversas culturas, em grupos com patologias identificadas e em diferentes faixas etárias.

Pela importância da funcionalidade manual torna-se pertinente compreender o desenvolvimento do comportamento assimétrico, quer ao nível da preferência quer da proficiência e funcionalidade. O objectivo do presente ensaio consubstancia-se na descrição do desenvolvimento da preferência e proficiência manual relacionadas com a idade e com o género, e sua caracterização em condições patológicas.

\section{ASSIMETRIAS MANUAIS: DA CONCEPÇÃO AOS PRIMEIROS 3 ANOS DE VIDA}

A tendência para a preferência manual direita, típica da população adulta, parece estar já presente no início da vida. Apesar da preferência manual não ser uma característica óbvia de crianças pequenas, tornando-se mais evidente com o início da escolaridade, podem observar-se algumas tendências de preferência mesmo antes do nascimento. As assimetrias posturais espontâneas de fetos e bebés recém-nascidos têm sido estudadas na perspectiva de explicação de tendências posteriores de desenvolvimento. Os resultados mostram que entre as 10 e as 12 semanas de vida já é possível observar movimentos lateralizados dos membros e cabeça e que a tendência para a direita é predominante(13). Alguns aspectos e tendências verificados durante o período pré-natal têm sido relacionados com características comportamentais pós-parto. Por exemplo, existe alguma indicação de que os fetos que sugaram o seu polegar direito durante a gestação, quando examinados entre o segundo e quarto dias após o seu nascimento, viravam a cabeça para o lado direito(1). A preferência para o lado direito na sucção do polegar às 15 semanas de gestação também foi recentemente relacionada com a preferência manual direita aos 10-12 anos de idade(4). Porém, esta preferência estável para a direita não foi sempre evidente. De Vries et al.(5) observaram os contactos da mão com a face efectuados por fetos com idade gestacional entre 12 e 38 semanas relacionando-os com a posição preferencial da cabeça após o nascimento. Contudo, não foi possível suportar a associação entre contactos da mão e posição da cabeça na formação de uma sinergia ipsilateral estável e preferencial. Por outro lado, a posição da cabeça durante o período fetal e o virar da cabeça espontâneo logo após o nascimento foram relacionados com actividades manuais (manipulação e preferência) na infância(6).

A observação de recém-nascidos demonstra, do mesmo modo, uma variedade de tendências motoras laterais, nos contactos da mão com a face, na orientação da cabeça $(7,8)$, no reflexo de preensão $(9,10)$, no reflexo do caminhar $(8,11)$, ou no reflexo tónico assimétrico e contralateral do pescoço(11). Porém, a maioria dos estudos observa as tendências laterais após o nascimento ao nível do tronco e membros superiores por serem um potencial indicativo da preferência manual adoptada posteriormente. Os estudos que focam as assimetrias laterais no reflexo do caminhar como indicativo da preferência lateral dos membros inferiores são muito menos frequentes e não revelam tendências assimétricas.

À medida que vão crescendo, os bebés mostram preferência manual em acções como apontar, agarrar e manipular objectos, traduzida pela frequência do uso de cada mão. A idade dos 3 anos parece constituir um marco no uso preferencial por uma das mãos, normalmente a direita ${ }^{(12)}$. Alguns factores podem influenciar estas flutuações, tais como as mudanças no desenvolvimento da organização e controlo dos movimentos do braço e mão, controlo postural, associação a comportamentos locomotores, ou a constrangimentos da tarefa. Não obstante, à medida que a idade avança torna-se notória a preferência consistente por um dos lados, com uma grande variabilidade no que diz respeito ao processo de desenvolvimento da preferência manual durante o primeiro ano de vida ${ }^{(13,16)}$. Esta variabilidade pode ser real ou apresentar alguma contaminação metodológica, pela dificuldade de avaliação típica destas idades. Após os 12 meses, os autores são unânimes em considerar uma certa estabilidade ao nível dos com- 
portamentos de preferência manual, sobretudo quando se trata da preferência sobre a mão direita, como acontece em cerca de $90 \%$ dos casos. As mudanças no desenvolvimento da preferência manual durante os primeiros anos de vida parecem estar associadas a reorganizações sucessivas do sistema motor, que ocorrem à medida que a criança aprende a sentar, gatinhar e andar, assim como às novas possibilidades de acção sobre o envolvimento. Como tal, o desenvolvimento da preferência manual afigura-se como altamente maleável e sensível a uma diversidade de novas experiências sensorio-motoras $(17,18)$, pelo que os primeiros movimentos assimétricos são muito flexíveis e sujeitos a uma oscilação e instabilidade antes de se estabelecerem numa determinada direcção. A determinação destas tendências é ainda insatisfatória, pela escassez de estudos longitudinais. Existe alguma controvérsia sobre a idade de estabilização da preferência manual. Alguns autores defendem a idade dos 3 anos(19), outros dos 4 anos(20) e outros ainda sugerem que, apesar da preferência manual estar estabelecida por volta dos 5 ou 6 anos, esta pode sofrer alterações até cerca dos 13 anos, fruto das pressões sociais ${ }^{(21)}$. A consistência da preferência, difícil de medir com objectividade, parece aumentar com a idade, ao mesmo tempo que se fixa uma preferência definida.

Apesar de muitos estudos procurarem referenciar idades, com evidente efeito normativo, o próprio conceito de estabilidade é pouco preciso, o que pode reflectir-se no sentido das conclusões dos estudos. Algumas investigações descrevem uma tendência para a direita mais elevada no sexo masculino comparativamente ao sexo feminino no que diz respeito à rotação da cabeça $(7,22)$ e à força de preensão palmar(23), enquanto outras apresentam resultados contrários $(24,25)$. A tendência para uma preferência manual direita parece ser mais precoce no sexo feminino do que no masculino no que diz respeito ao comportamento de agarrar objectos (26) o que sugere um desenvolvimento da lateralização cerebral mais precoce nas meninas do que nos meninos, hipotetizando a possibilidade deste resultado ser um precursor da assimetria mais acentuada nas mulheres adultas comparativamente aos homens.

Uma porção significativa de bebés recém-nascidos demonstra reflexos e movimentos espontâneos mais fortes e mais coordenados para o lado direito do corpo do que para o lado esquerdo. Porém, uma preferência para a esquerda superior ao normal tem sido observada consistentemente em prematuros (14, 27-30), em especial nos rapazes ${ }^{(27,29)}$. Outros factores relacionados com o bebé ou com o momento do parto têm sido ligados a uma percentagem elevada de preferência manual esquerda ou mista. Entre eles encontram-se, o baixo peso, ordem elevada de fratria, dificuldades respiratórias, trabalho de parto prolongado, tipo de parto (cesariana, uso de fórceps, apresentação pélvica), e incompatibilidade $\mathrm{Rh}^{(31,32)}$. Para além disso, alguns factores relacionados com a mãe, como idade precoce ou elevada da mãe no momento do nascimento do bebé, sintomas de depressão, ansiedade, consumo de drogas, tabaco ou álcool por parte da mãe durante a gravidez, podem influenciar a trajectória típica das assimetrias funcionais dos seus filhos, traduzindo-se numa maior prevalência de preferência manual mista ou para a esquerda(32-34). Estes resultados sugerem que as origens neuro-desenvolvimentais da preferência manual podem estar parcialmente ligadas ao ambiente químico a que o feto está exposto no útero, fomentado pelos vários factores de stress pré-natal mencionados anteriormente.

\section{ASSIMETRIAS MANUAIS: PERÍODO PRÉ-ESCOLAR E ESCOLAR}

Os avanços no controle motor durante o período préescolar, os quais dependem tanto da maturação cerebral e física como do refinamento das competências através da experiência, permitem o estudo da preferência manual em acções como desenhar, cortar com uma tesoura, apanhar um objecto do chão, ou lançar um objecto a um alvo. Muitos estudos investigaram a preferência manual de crianças dos 3 aos 12 anos de idade na execução de várias acções como as descritas anteriormente(e.g. 19, 35) bem como, a assimetria manual em vários testes de performance(e.g. 36, 37). Merece ser realçado que, apesar das medidas de performance permitirem distinções precisas numa escala quantitativa, elas não são, em parte, acessíveis a crianças até à idade pré-escolar nem a crianças com perturbações do desenvolvimento ou limitações sensoriais, físicas e cognitivas. Os resultados dos estudos apontam para uma tendência das populações pré-escolar e 
escolar para uma preferência manual direita(38, 39), com uma percentagem de sinistrómanos semelhante à da população adulta (aproximadamente 10\%). Porém, tal como na população adulta, essa tendência parece variar com a cultura onde está inserida a criança. Por exemplo, Fagard e Dahmen ${ }^{(40)}$ verificaram que, entre os 5 e os 9 anos, $16.7 \%$ das crianças Francesas usam a mão esquerda na escrita, por comparação com $3.3 \%$ das crianças Tunisinas, e o estudo de Holder e Kateeba (41) registou, no Uganda, $4.8 \%$ de crianças sinistrómanas entre os 4 e os 19 anos.

Pode existir um forte efeito da cultura, tradições, tolerância e estereótipos comportamentais na tendência biológica para uma preferência determinada. Viviani ${ }^{(42)}$ verificou, em crianças italianas entre os 5 e os 11 anos, que as do meio rural apresentavam percentagens significativamente inferiores de preferência manual esquerda $(6.2 \%)$ relativamente às do meio urbano $(20.1 \%)$.

No que diz respeito ao desenvolvimento da preferência manual neste período, os investigadores concordam que não é a direcção que muda com a idade ${ }^{(43}$, 44) mas sim a consistência (intensidade) da preferência manual $(45,46)$. Por exemplo, Bryden, Roy e Spence ${ }^{(45)}$ verificaram que as crianças mais jovens (entre os 3 e 5 anos) eram menos consistentes do que as mais velhas (a partir dos 7 anos) e do que os adultos. Também Greenwood et al.(47) observaram, em 5000 crianças Irlandesas dos 3 aos 18 anos, que as mais novas e os rapazes demonstraram uma frequência e intensidade menos elevadas na tendência para a direita. Porém, Ounsted et al.(48), num estudo longitudinal em que avaliaram 199 crianças aos 2, aos 4 e aos $7 \frac{1}{2}$ anos, verificaram que a preferência manual foi constante ao longo deste período. Das crianças destrímanas aos $7 \frac{1}{2}$ anos, mais de $70 \%$ demonstraram a mesma preferência manual manifestada aos 2 anos e $89 \%$ manteve a mesma preferência revelada aos 4 anos. Para as crianças sinistrómanas, os valores foram idênticos aos das destrímanas, ou seja $76 \%$ aos 2 anos e $86 \%$ aos 4 anos. No sentido de avaliar a consistência da preferência manual, McManus et al.(49) verificaram, numa amostra de 314 crianças com idades de 3, 4, 5 e 7 anos, que a direcção da preferência parece estar determinada aos 3 anos, e que a consistência no uso da mão preferida aumentou ao longo do tempo, mais rapidamente nas crianças sinistrómanas. Estas parecem demonstrar uma preferência manual mais fraca do que as destrímanas aos 3 anos, mas de similar consistência aos 7 anos. Resultado semelhante com crianças sinistrómanas foi observado por Bryden e Mayer(50). $\mathrm{O}$ aumento da consistência na preferência manual direita com a idade não tem ainda uma explicação clara. Presumivelmente poderá ser o resultado das interacções sociais e da adaptação a um mundo "orientado à direita", mas poderá ser admitida uma regulação ao nível maturacional, que decorrerá mesmo na ausência de quaisquer influências do meio. Recentemente Dubois et al.(51) observaram em bebés entre o primeiro e o quarto mês de vida marcadores estruturais de assimetrias hemisféricas, ou seja, diferenças inter-hemisféricas na proporção de neurónios no tracto cortico-espinal, sendo esta assimetria mais evidenciada para o lado esquerdo. Apesar de este estudo não clarificar se estas assimetrias estruturais são a causa ou consequência do desenvolvimento das assimetrias funcionais, os autores sugerem que esta organização neural está relacionada com o desenvolvimento posterior da lateralização funcional. Provavelmente será a interacção destes e de outros factores alguns dos aspectos que actuam sinergeticamente no sentido de intensificar o uso da mesma mão ao longo da vida.

O conhecimento sobre a assimetria manual, muito dependente das tarefas utilizadas, não reúne consensos fáceis. Enquanto em alguns estudos(e.g. 52, 53) não se observaram diferenças estatisticamente significativas ao longo da idade, em outros (e.g. 45, 54) verificou-se uma maior assimetria manual nos grupos mais jovens. Mas Bryden et al.(55), por exemplo, verificaram, na tarefa motora WatHand Cabinet Test, que os sinistrómanos apresentavam maior variação do que os destrímanos nas assimetrias manuais ao longo da idade, e que as crianças mais jovens demonstravam um grau de lateralização mais fraco do que as crianças mais velhas e do que os adultos. Para além disso, tem sido observada uma assimetria funcional menos acentuada nos sinistrómanos(56), facto que tem sido explicado pelo não uso da sua mão dominante em muitas tarefas da vida diária traduzido pela vivência num mundo destro. Para além da idade, a lateralidade manual parece também estar de algum modo associada ao sexo. Enquanto alguns autores não documentam diferen- 
ças significativas entre raparigas e rapazes(e.g. 19, 57), outros observam uma percentagem superior de sinistrómanos no sexo masculino(e.g. 41, 58).

Em relação à consistência (intensidade) da preferência manual, existe uma incidência superior de ambidestralidade no sexo masculino(59) enquanto o sexo feminino revela maior tendência para intensificar o uso da mão preferida, no caso de esta ser a direita. De acordo com alguns autores (e.g. 60, 61), a intensificação superior no sexo feminino, relativamente ao masculino, devese à maior pressão cultural a que as mulheres estão sujeitas desde muito novas. A confirmar este facto, Porac e Coren (62) observaram uma taxa superior de sucesso no sexo feminino relativamente à mudança para uma preferência manual direita.

No que respeita à performance, alguns estudos reportam diferenças entre os sexos no grau de assimetria manual, sendo o sexo feminino mais fortemente lateralizado do que o masculino no desempenho de algumas tarefas motoras ${ }^{(63,64)}$. Pedersen et al.(64), por exemplo, avaliaram 112 crianças com idades compreendidas entre os 7 e os 9 anos, utilizando duas tarefas do Movement Assessement Battery for Children (atirar pequenas bolas e fazer um jogo de encaixe simples). O estudo pretendeu avaliar as diferenças entre os sexos nas habilidades manuais. Os resultados revelaram que, na tarefa das bolas, o sexo feminino mostrou ser mais assimétrico, enquanto na tarefa de encaixe, não se verificaram diferenças entre os sexos. Os autores sugeriram que as diferenças entre os sexos, a existirem, parecem ser específicas de algumas tarefas, o que reforça o papel de eventuais condicionamentos sociais na expressão de preferências laterais. A especificidade da tarefa no que diz respeito à assimetria manual e sua relação com as diferenças entre os sexos foram também corroboradas por outros autores $(65,66)$.

Algumas condições atípicas, como o autismo(e.g. 67), a esquizofrenia(e.g. 68), as doenças do sistema imunitário(e.g. 69) ou desordens desenvolvimentais(e.g. 70, 71) têm sido associadas a padrões atípicos de lateralização. O modelo patológico, sugerido por Satz et al. ${ }^{(72)}$, postula que a preferência manual esquerda é determinada tanto geneticamente, resultando numa preferência manual esquerda natural, como patologicamente. Do ponto de vista dos autores, o segundo caso é consequência de danos cerebrais prematuros, tendo-se baseado, para esta sugestão, na observação de um elevado número de sinistrómanos em sujeitos com danos cerebrais ou com suspeita da existência desses danos. Porém, parece que é a ausência de uma tendência lateral ou uma assimetria menos acentuada para a direita que caracteriza estas populações patológicas, ao invés de uma percentagem mais elevada de sinistrómanos bem lateralizados (e.g. $73,74)$. Recentemente este assunto tem suscitado interesse, talvez pela exuberância de estudos associados às desordens coordenativas na criança(para uma revisão exaustiva sobre este assunto ver 75 ).

\section{ASSIMETRIAS MANUAIS: ADULTOS E IDOSOS}

As tendências manuais em adultos são, tal como em crianças em idade escolar, analisadas em relação à preferência (a auto-definição, os questionários e as tarefas motoras unilaterais) e à proficiência (testes de performance). No entanto, Peters(76) recomenda que, em idades superiores a 40 anos, os sujeitos não devam ser inquiridos sobre a sua preferência manual com base apenas na mão usada na escrita. Esta recomendação baseia-se na pressão social a que possam ter estado sujeitos no caso de uma preferência inicial pelo uso da mão esquerda, sendo vários os estudos que justificam o cuidado desta advertência(e.g. 77, 78). É importante referir que, apesar da diminuição da pressão social sobre o uso da mão esquerda, assistese ainda a alguma estigmatização quando esta é a preferida em detrimento da direita para as várias tarefas da vida diária, entre as quais se destaca claramente a escrita ${ }^{(42,47)}$.

Os estudos que analisam a preferência manual ao longo da vida demonstram que a percentagem de sinistrómanos diminui com o avançar da idade, sendo drasticamente sub-representada nos idosos(7982). Por exemplo, Bryden, Bulman-Fleming e MacDonald( ${ }^{(80)}$ descreveram, numa amostra de três gerações, uma percentagem de sinistrómanos de $1.9 \%$ nos indivíduos com idade superior a 64 anos, de $8.6 \%$ com idades compreendidas entre os 35 e os 54 anos, e de $19.8 \%$ com idades entre os 20 e os 29 anos. O aumento da percentagem de sinistrómanos nas gerações mais jovens tem sido atribuído a várias causas, entre as quais um relaxamento das atitudes sociais e uma maior permissão face ao uso da mão esquerda como mão preferida $(83,84)$. Também as 
hipóteses de acumulação de pressões num mundo construído para destrímanos ${ }^{(60)}$, ou uma expectativa de vida mais reduzida nos sinistrómanos, devido a uma incidência mais elevada de morte não natural( ${ }^{85}$, 86), têm merecido alguma atenção.

Coren e Halpern(85) agregaram as várias explicações para o declínio da preferência manual esquerda com a idade em duas grandes hipóteses: a hipótese da eliminação e a hipótese da modificação. A primeira sugere que os sinistrómanos encontram mais dificuldades ao nível físico por viverem num mundo destro, estando, por conseguinte, mais sujeitos a sofrerem acidentes e, para além disso, mais propensos a riscos relacionados com a saúde tendo, portanto, um tempo de vida mais curto. A segunda hipótese, a da modificação, expressa que as pressões para modificar os comportamentos da preferência manual diferem entre gerações, sendo mais flexíveis actualmente. Ou seja, a hipótese da eliminação remete para factores mais biológicos, enquanto a da modificação sugere factores mais sociais e históricos.

A hipótese da eliminação tem demonstrado resultados contraditórios. Enquanto alguns estudos não a confirmam (87, 88), apresentando até resultados contrários, indicando uma vantagem não significativa de sobrevivência mais elevada nos sinistrómanos(e.g. 87), outros relatam uma frequência mais elevada nos sinistrómanos de acidentes( ${ }^{(89)}$, de ferimentos acidentais $(90,91)$, de quedas $(92,93)$ e, mais recentemente, de doenças cardíacas ${ }^{(94)}$, aumentando a vulnerabilidade à morte acidental.

Por outro lado, investigações que utilizaram um critério de classificação da preferência manual mais abrangente (não considerando apenas a classificação dicotómica, em sinistrómanos e destrímanos mas um critério com base na consistência, isto é, na intensidade da preferência, para além da direcção) observaram serem os sinistrómanos não consistentes (menos lateralizados), relativamente aos outros grupos, os mais propícios a um risco mais acentuado de morte prematura(95) e de acidentes ${ }^{(93)}$.

A hipótese da modificação sustenta que a maior ou menor pressão exercida pelas sociedades e culturas sobre os sinistrómanos resulta numa modificação dos seus comportamentos constrangendo-os a agir como destrímanos, facto confirmado por alguns estudos ${ }^{(77,}$ 96) e contestado por outros ${ }^{(97)}$. Porém, esta hipótese não explica completamente a reduzida incidência de sinistrómanos em indivíduos idosos, uma vez que as tentativas para mudar o uso da mão são muito específicas a determinadas tarefas, tais como comer e escrever e, mesmo quando efectivas, essas mudanças não tendem a produzir um efeito generalizado(61, 98). Dos diversos estudos apresentados conclui-se as duas hipóteses não são mutuamente exclusivas. Contudo, o debate está longe de terminar, permanecendo ainda por desvendar a verdadeira razão da reduzida prevalência de sinistrómanos na população mais idosa.

A intensidade da preferência manual ao longo da idade também tem sido objecto de investigação (99, 100). Teixeira ${ }^{(100)}$, por exemplo, analisou este aspecto em destrímanos divididos em três grupos (20, 40 e 60 anos), tendo detectado um efeito significativo através dos grupos de idade, nomeadamente no que respeita à comparação entre o grupo com 60 anos e o grupo com 20 anos. De igual forma, num trabalho não publicado, Rodrigues, Lamboglia, Cabral, Barreiros e Vasconcelos(101) analisaram a intensidade da preferência manual em 1977 destrímanos e sinistrómanos, de ambos os sexos, distribuídos por cinco grupos de idade (6-11 anos, 12-18 anos, 19-33 anos e 56-95 anos). Os resultados demonstraram diferenças significativas entre os grupos, porém, o efeito da idade não foi similar em sinistrómanos e destrímanos. Nestes últimos, verificou-se que as crianças exibiram um grau de preferência mais fraco do que os outros grupos de idade, sendo o grupo dos idosos o mais fortemente lateralizado. Este padrão não foi evidente nos sinistrómanos, onde apenas o grupo de adolescentes diferiu significativamente dos outros grupos, demonstrando uma fraca lateralização. É de salientar, contudo, que apesar dos outros grupos não diferirem entre si significativamente, os adultos demonstraram uma lateralização mais acentuada, seguida das crianças e dos idosos. Os resultados obtidos no grupo dos destrímanos parecem corroborar os efeitos da prática e da experiência, relacionados com um ambiente que favorece o uso da mão direita. Estes factores têm sido apontados como determinantes no aumento da intensidade da preferência manual ao longo da idade. Esta justificação não se aplica, no entanto, aos resultados observados nos grupos de sinistrómanos, os quais não revelaram uma tendência clara com a idade. 
No que respeita ao efeito da idade na proficiência manual, não há consenso entre os estudos. Assim, enquanto algumas investigações reportam uma diminuição da assimetria manual com o avançar da idade(e.g. 102, 103), outros mencionam que a assimetria permanece inalterada com a idade(e.g. 37, 104) e, outros ainda, que a assimetria manual ao longo da idade depende do tipo de tarefas usadas bem como da sua complexidade(e.g. 100, 105). As alterações na assimetria manual com a idade têm sido frequentemente apontadas como uma adaptação compensatória às mudanças do processamento neural relacionadas com a idade. Duas hipóteses têm sido propostas para explicar este aspecto: a hipótese diferencial da idade(106) e a hipótese da redução da assimetria hemisférica em idosos(107). De acordo com a primeira hipótese, com o avançar da idade assiste-se a um declínio mais acentuado das funções do hemisfério direito relativamente ao hemisfério esquerdo. A segunda hipótese propõe que o envelhecimento conduz a um padrão simétrico bilateral das funções hemisféricas. Estas duas hipóteses foram testadas por Hausmann, Gunturkun e Corballis (108) em tarefas que exigiam processamento específico de cada hemisfério. Os resultados demonstraram que as mudanças nas assimetrias hemisféricas relativas à idade são diferentes para tarefas com processamento específico do hemisfério direito e do esquerdo, não suportando nenhuma das hipóteses. Por conseguinte, a inconsistência dos resultados dos estudos atrás mencionados pode estar relacionada com o tipo de tarefa.

No que diz respeito ao efeito do sexo na preferência manual em adultos e idosos, os resultados, tal como nos outros momentos descritos, também se apresentam inconclusivos. Enquanto alguns estudos documentam a ausência de diferenças significativas entre sexos $(109,110)$, outros observaram uma percentagem de homens sinistrómanos superior à de mulheres $(82$, 111). Um estudo recente de meta-análise sobre esta questão, efectuado por Sommer et al.(112), confirmou esta tendência. A explicação que admite mais consenso, nesta linha de pesquisa que sugere uma maior percentagem de homens sinistrómanos relativamente às mulheres sinistrómanas, é a de que os homens são, por um lado, menos sujeitos às pressões culturais e, por outro, mais resistentes a essas pressões no sentido da mudança para uma preferên- cia manual direita $(47,113)$. Contudo, outras explicações têm sido apresentadas, nomeadamente as relacionadas com a contribuição genética $(114,115)$, as quais postulam que a expressão do gene que contribui para a preferência manual para a direita é mais acentuada no sexo feminino do que no sexo masculino. Esta relação parece ser corroborada por estudos cujos resultados revelaram uma associação entre os cromossomas sexuais e a preferência manual, ou seja, o gene associado com a preferência manual

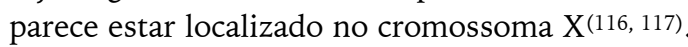
Como os genes recessivos no cromossoma X são expressos mais frequentemente no sexo masculino do que no sexo feminino, esta explicação pode considerar-se plausível no que diz respeito à maior frequência de indivíduos do sexo masculino com preferência manual esquerda.

Por fim, temos as interpretações relativas à contribuição hormonal(118), sugerindo que os níveis de testosterona, mais elevados no sexo masculino comparativamente ao feminino, contribuem para o desenvolvimento da lateralização cerebral, podendo induzir um atraso no desenvolvimento do hemisfério esquerdo e, consequentemente, resultar numa expressão manual direita menos pronunciada nos homens. Contudo, embora alguns estudos efectuados sobre a relação entre a exposição no útero a hormonas esteróides e a prevalência de uma preferência manual não destra pareçam corroborar esta teoria $(119$, 120), uma meta-análise realizada recentemente aponta para resultados contrários ${ }^{(121)}$.

No que respeita às medidas de performance, têm sido documentadas por vários autores diferenças significativas entre os sexos, com os homens menos lateralizados do que as mulheres na assimetria motora funcional $(37,122)$. Contudo, há relatos de outros estudos onde não se observaram diferenças significativas entre os sexos nesta variável(123-125). Não obstante, apesar das diferenças não se revelarem significativas em relação à assimetria manual, Lissek et al.(123) observaram diferenças na activação cerebral durante a realização da tarefa motora, sendo que o sexo feminino demonstrou uma activação cortical mais bilateral do que o sexo masculino. A natureza da tarefa bem como a organização cerebral distinta em ambos os sexos têm sido apontadas como os principais factores que contribuem para esta discrepância de resultados ${ }^{(112,124)}$. 
Na população adulta também se tem estudado a relação da preferência manual esquerda ou mista com patologias. Existem estudos associando a preferência manual esquerda a condições físicas e mentais, tais como, apneia do sono(126), asma ${ }^{(127)}$, doença de Alzheimer(128), síndrome de Williams-Beuren ${ }^{(129)}$, psi$\operatorname{cose}^{(130)}$, esquizofrenia(131). Os danos no hemisfério esquerdo relacionados com as patologias são apontados pelos autores como possível causa da frequência mais elevada de sinistrómanos nestes grupos.

\section{CONCLUSÃO}

As evidências provenientes de vários estudos mostram que as assimetrias laterais variam ao longo do desenvolvimento, com um faseamento algo previsível, mas com algumas incoerências ainda por explicar. O sexo, o estado neurológico do sujeito e o tipo de sociedade em que este está inserido são factores que parecem interferir no desenvolvimento da preferência manual e na consequente assimetria manual. Pode-se assim dizer que a assimetria resulta de factores biológicos, sociais e culturais característicos de um determinado espaço geográfico e de uma contextualização histórica e cultural. Para além disso, deve referir-se que existe grande variabilidade de metodologias e procedimentos (no que respeita ao número e tipo de instrumentos aplicados para a classificação da preferência manual, ao próprio critério de classificação dos grupos de preferência manual e ao tipo de testes de avaliação da proficiência manual que permitem ajuizar a consequente assimetria funcional). Existem ainda as diferenças amostrais entre estudos, nomeadamente ao nível sócio-cultural, como foi referido. Todas estas questões dificultam a comparação dos resultados. Consequentemente, a posição teórica de cada autor e a sua predilecção por um método ou por outro, resultam numa diversidade de nuances sobre a conclusão mais geral mas inquestionável de que a preferência manual, a proficiência manual e a consequente assimetria manual variam e sofrem oscilações ao longo do desenvolvimento do sujeito.

\author{
CORRESPONDÊNCIA \\ Paula Cristina Rodrigues \\ Rua Dr. José Marinho, nº 267, 4460-752 Custóias, \\ Matosinhos \\ Portugal; \\ Telefone: +35122 9545300; +351918808119 \\ E-mail: packn2@gmail.com
}




\section{REFERÊNCIAS}

1. Hepper PG, Shahidullah S, White R (1991). Handedness in the human fetus. Neuropsychologia, 29(11): 1107-11

2. Ververs IA, de Vries JI, van Geijn HP, Hopkins B (1994). Prenatal head position from 12-38 weeks. II. The effects of fetal orientation and placental localization. Early Hum Dev, 39(2): 93-100

3. McCartney G, Hepper P (1999). Development of lateralized behaviour in the human fetus from 12 to 27 weeks' gestation. Dev Med Child Neurol, 41(2): 83-6

4. Hepper PG, Wells DL, Lynch C (2005). Prenatal thumb sucking is related to postnatal handedness. Neuropsychologia, 43(3): 313-5

5. de Vries JI, Wimmers RH, Ververs IA, Hopkins B, Savelsbergh GJ, van Geijn HP (2001). Fetal handedness and head position preference: a developmental study. Dev Psychobiol, 39(3): 171-8

6. Tachibana H, Iwasa S (2001). [Relationships among the position in the fetal period, head-turning during three days after birth, and hand activity in infancy]. Shinrigaku Kenkyu, 72(3): 177-85

7. Beuter CR, Pedroso FS, Mazetto RC, Santos CT, Rossi AG (2007). Association between dynamic asymmetry of the newborn's head and intrauterine factors. Arq Neuropsiquiatr, 65(2A): 218-21

8. Domellof E, Hopkins B, Ronnqvist L (2005). Upper and lower body functional asymmetries in the newborn: do they have the same lateral biases? Dev Psychobiol, 46(2): 133-40

9. Tan U, Tan M (1999). Incidences of asymmetries for the palmar grasp reflex in neonates and hand preference in adults. Neuroreport, 10(16): 3253-6

10. Thompson AM, Smart JL (1993). A prospective study of the development of laterality: neonatal laterality in relation to perinatal factors and maternal behavior. Cortex, 29(4): 649-59

11. McCormick CM, Maurer DM (1988). Unimanual hand preferences in 6-month-olds: consistency and relation to familial-handedness. Infant Behav Dev, 11(1): 21-29

12. Hinojosa T, Sheu CF, Michel GF (2003). Infant hand-use preferences for grasping objects contributes to the development of a hand-use preference for manipulating objects. Dev Psychobiol, 43(4): 328-34

13. Michel GF, Tyler AN, Ferre C, Sheu CF (2006). The manifestation of infant hand-use preferences when reaching for objects during the seven-to thirteen-month age period. Dev Psychobiol, 48(6): 436-43

14. Ronnqvist L, Domellof E (2006). Quantitative assessment of right and left reaching movements in infants: a longitudinal study from 6 to 36 months. Dev Psychobiol, 48(6): 444-59

15. Stroganova TA, Pushina NP, Orekhova EV, Posikera IN, Tsetlin MM (2004). [Functional brain asymmetry and individual differences in hand preference in early ontogenesis]. Fiziol Cheloveka, 30(1): 20-30

16. 16.Rice T, Plomin R, De Fries JC (1984). Development of hand preference in the Colorado Adoption Project. Percept Mot Skills, 58(3): 683-9

17. Corbetta D, Williams J, Snapp-Childs W (2006). Plasticity in the development of handedness: evidence from normal development and early asymmetric brain injury. Dev Psychobiol, 48(6): 460-71

18. Corbetta D, Thelen E (1999). Lateral biases and fluctuations in infants' spontaneous arm movements and reaching. Dev Psychobiol, 34(4): 237-55.

19. Longoni AM, Orsini L (1988). Lateral preferences in preschool children: a research note. J Child Psychol Psychiatry, 29(4): 533-9

20. Teixeira LA, Gasparetto ER (2002). Lateral asymmetries in the development of the overarm throw. J Mot Behav 34(2): 151-160.

21. Ozturk C, Durmazlar N, Ural B, Karaagaoglu E, Yalaz K, Anlar B (1999). Hand and eye preference in normal preschool children. Clin Pediatr (Phila), 38(11): 677-80

22. Ronnqvist L, Hopkins B (1998). Head position preference in the human newborn: a new look. Child Dev, 69(1): 1323

23. Tan U, Tan M (2001). Testosterone and grasp-reflex differences in human neonates. Laterality, 6(2): 181-192

24. Tan U (2000). Grasp-reflex in Human Neonates: Distribution, Sex Difference, Familial Sinistrality, and Testosterone. Side Bias: A Neuropsychological Perspective. In MK Mandal, MB Bulman-Fleming,G Tiwari. Dordrecht: Kluwer Academic Publishers, 63-82

25. Tan U, Ors R, Kurkcuoglu M, Kutlu N, Cankaya A (1992). Lateralization of the grasp reflex in male and female human newborns. Int J Neurosci, 62(3-4): 155-63

26. Humphrey D, Humphrey G (1987). Sex differences in infant reaching. Neuropsychologia, 25(6): 971-975

27. Grattan MP, Nelson MN, White-Traut RC, Vasan U, Gu GG, Littau S (2005). Sex differences in high-risk premature infants' asymmetric movement development. Phys Occup Ther Pediatr, 25(4): 5-28

28. O'Callaghan MJ, Burn YR, Mohay HA, Rogers Y, Tudehope DI (1993). The prevalence and origins of left hand preference in high risk infants, and its implications for intellectual, motor and behavioural performance at four and six years. Cortex, 29(4): 617-27

29. Samsom JF, de Groot L, Cranendonk A, Bezemer D, Lafeber HN, Fetter WP (2002). Neuromotor function and school performance in 7-year-old children born as highrisk preterm infants. J Child Neurol, 17(5): 325-32

30. Ross G, Lipper E, Auld PA (1992). Hand preference, prematurity and developmental outcome at school age. Neuropsychologia, 30(5): 483-94

31. McKeever WF, Suter PJ, Rich DA (1995). Maternal age and parity correlates of handedness: gender, but no parental handedness modulation of effects. Cortex, 31(3): 543-53

32. Bailey LM, McKeever WF (2004). A large-scale study of handedness and pregnancy/birth risk events: implications for genetic theories of handedness. Laterality, 9(2): 175-88

33. Rodriguez A, Waldenstrom U (2008). Fetal origins of child non-right-handedness and mental health. J Child Psychol Psychiatry, 49(9): 967-76

34. Glover V, O'Connor TG, Heron J, Golding J (2004). Antenatal maternal anxiety is linked with atypical handedness in the child. Early Hum Dev, 79(2): 107-18

35. Giagazoglou P, Fotiadou E, Angelopoulou N, Tsikoulas J, Tsimaras V (2001). Gross and fine motor skills of left-handed preschool children. Percept Mot Skills, 92(3 Pt 2): 1122-8

36. Bryden PJ, Roy EA (2005). Unimanual performance across the age span. Brain Cogn, 57(1): 26-9

37. Doyen AL, Dufour T, Caroff X, Cherfouh A, Carlier M (2008). Hand preference and hand performance: cross-sec- 
tional developmental trends and family resemblance in degree of laterality. Laterality, 13(2): 179-97

38. Gabbard C, Helbig CR (2004). What drives children's limb selection for reaching in hemispace? Exp Brain Res, 156(3): 325-32.

39. Mahone EM, Wodka EL, Hiemenz JR (2006). Hand and eye preference and their association with task approach by preschoolers. Percept Mot Skills, 102(3): 691-702

40. Fagard J, Dahmen R (2004). Cultural influences on the development of lateral preferences: a comparison between French and Tunisian children. Laterality 9(1): 67-78

41. Holder MK, Kateeba D (2004). Hand preference survey of 5136 school children in Western Uganda. Laterality, 9(2): 201-7

42. Viviani F (2006). Insights on behavioural and educational pressures on laterality development in children. Papers on Anthropology XV: 294-301

43. Cavill S, Bryden P (2003). Development of handedness: comparison of questionnaire and performance-based measures of preference. Brain Cogn, 53(2): 149-51

44. Annett M (2004). Hand preference observed in large healthy samples: classification, norms and interpretations of increased non-right-handedness by the right shift theory. Br J Psychol, 95(Pt 3): 339-53

45. Bryden PJ, Roy EA, Spence J (2007). An observational method of assessing handedness in children and adults. Dev Neuropsychol, 32(3): 825-46

46. Singh M, Manjary M, Dellatolas G (2001). Lateral preferences among indian school children. Cortex, 37(2): 231-41

47. Greenwood JG, Greenwood JJ, McCullagh JF, Beggs J, Murphy CA (2007). A survey of sidedness in Northern Irish schoolchildren: the interaction of sex, age, and task. Laterality, 12(1): 1-18

48. Ounsted M, Cockburn J, Moar VA (1985). Hand preference: its provenance, development, and associations with intellectual ability at the age of 7.5 years. J Dev Behav Pediatr, 6(2): 76-80

49. McManus C, Sik G, Cole DR, Mellon AF, Wong J, Kloss J (1988). The development of handedness in children. Br J Dev Psychol, 6: 257-273

50. Bryden MP, Mayer M (2008). Hand preference and performance abilities in children and adults. Brain Cogn, 67 (supplement 1): S15

51. Dubois J, Hertz-Pannier L, Cachia A, Mangin JF, Le Bihan D, Dehaene-Lambertz G (2009). Structural asymmetries in the infant language and sensori-motor networks. Cereb Cortex, 19(2): 414-23

52. Dellatolas G, De Agostini M, Curt F, Kremin H, Letierce A, Maccario J, Lellouch J (2003). Manual skill, hand skill asymmetry, and cognitive performances in young children. Laterality, 8(4): 317-38

53. Nunes G, Braga LW, Rossi L, Lawisch VL, Nunes LG, Dellatolas G (2008). Hand skill assessment with a reduced version of the Peg Moving Task (PMT-5) in children: normative data and application in children with cerebral palsy. Arch Clin Neuropsychol, 23(1): 87-101

54. Roy EA, Bryden P, Cavill S (2003). Hand differences in pegboard performance through development. Brain Cogn, 53(2): 315-7

55. Bryden PJ, Roy EA, Spence J (2007). An observational method of assessing handedness in children and adults. Dev Neuropsychol, 32(3): 825-46.
56. Rousson V, Gasser T, Caflisch J, Jenni OG (2009). Neuromotor performance of normally developing left-handed children and adolescents. Hum Mov Sci, 28(6): 809-17

57. Fernandes D (2004). A mão, a preferência manual e a proficiência manual do idoso: estudo em idosos frequentadores de Centros de Dia. Porto: D. Fernandes. Dissertação de mestrado apresentada à Faculdade de Desporto da Universidade do Porto.

58. Faurie C, Vianey-Liaud N, Raymond M (2006). Do lefthanded children have advantages regarding school performance and leadership skills? Laterality, 11(1): 57-70.

59. Annett M, Kilshaw D (1983). Right- and left-hand skill II: Estimating the parameters of the distribution of L-R differences in males and females. Br J Psychol, 74 (Pt 2): 26983.

60. Porac C, Coren S (1981). Lateral preferences and human behavior. New York, Springer-Verlag.

61. Porac C (1996). Attempts to switch the writing hand: relationships to age and side of hand preference. Laterality, 1(1): 35-44.

62. Porac C, Coren S, Searleman A (1986). Environmental factors in hand preference formation: evidence from attempts to switch the preferred hand. Behav Genet, 16(2): 251-61.

63. Annett M (2002). Handedness and Brain Assymmetry: the right shift theory. Hove, UK, Psychology Press.

64. Pedersen AV, Sigmundsson H, Whiting HT, Ingvaldsen RP (2003). Sex differences in lateralisation of fine manual skills in children. Exp Brain Res, 149(2): 249-51

65. Largo RH, Fischer JE, Rousson V (2003). Neuromotor development from kindergarten age to adolescence: developmental course and variability. Swiss Med Wkly, 133(1314): 193-9

66. Dorfberger S, Adi-Japha E, Karni A (2009). Sex differences in motor performance and motor learning in children and adolescents: an increasing male advantage in motor learning and consolidation phase gains. Behav Brain Res, 198(1): 165-71

67. Hauck JA, Dewey D (2001). Hand preference and motor functioning in children with autism. J Autism Dev Disord, 31(3): 265-77

68. Collinson SL, Phillips TJ, James AC, Quested DJ, Crow TJ (2004). Is lateral bias anomalous in early-onset schizophrenia? Selected comparisons with normal populations. Psychiatry Res, 125(3): 219-24

69. Preti A, Lai A, Serra M, Zurrida GG (2008). Mixed handedness prevails among children and adolescents with infantile asthma and diabetes. Pediatr Allergy Immunol, 19(8): 769-72

70. Cairney J, Schmidt LA, Veldhuizen S, Kurdyak P, Hay J, Faught BE (2008). Left-Handedness and Developmental Coordination Disorder. Can J Psychiatry, 53(10): 696-699

71. Goez H, Zelnik N (2008). Handedness in patients with developmental coordination disorder. J Child Neurol, 23(2): 151-4

72. Satz P, Orsini DL, Saslow E, Henry R (1985). The pathological left-handedness syndrome. Brain Cogn, 4(1): 27-46

73. Leconte P, Fagard J (2006). Lateral preferences in children with intellectual deficiency of idiopathic origin. Dev Psychobiol, 48(6): 492-500

74. Van Strien JW, Lagers-Van Haselen GC, Van Hagen JM, De Coo IF, Frens MA, Van Der Geest JN (2005). Increased prevalences of left-handedness and left-eye sighting domi- 
nance in individuals with Williams-Beuren syndrome. $J$ Clin Exp Neuropsychol, 27(8): 967-76

75. Vasconcelos O, Rodrigues P, Barreiros J, Jacobsohn L (2009). Laterality, developmental coordination disorders and posture. Estudos em desenvolvimento motor II In LP Rodrigues, L Saraiva, J Barreiros, O Vasconcelos. Viana do Castelo: Escola Superior de Educação, Instituto Politécnico de Viana do Castelo, 19-26

76. Peters M (1998). Description and validation of a flexible and broadly usable handedness questionnaire. Laterality, 3(1): 77-96

77. Coude FX, Mignot C, Lyonnet S, Munnich A (2006). Discontinuity in the fall of left-handedness in a French population: a May '68 effect? Laterality, 11(1): 33-5

78. Searleman A, Porac C (2003). Lateral preference profiles and right shift attempt histories of consistent and inconsistent left-handers. Brain Cogn, 52 (2): 175-80

79. Van der Elst W, Van Boxtel MP, Van Breukelen GJ, Jolles J (2008). Is left-handedness associated with a more pronounced age-related cognitive decline? Laterality, 13(3): 234-54

80. Bryden PJ, Bulman-Fleming B, MacDonald V (1996). The measurement of handedness and its relation to neuropsychological issues. Manual asymmetries in motor performance. In D Elliot, E Roy. Florida: CRC Press, 57-76

81. Siengthai B, Kritz-Silverstein D, Barrett-Connor E (2008). Handedness and cognitive function in older men and women: a comparison of methods. J Nutr Health Aging, 12(9): 641-7

82. Suar D, Mandal MK, Misra I, Suman S (2007). Lifespan trends of side bias in India. Laterality, 12(4): 302-20

83. Porac C, Coren S, Duncan P (1980). Life-span age trends in laterality. J Gerontol 35(5): 715-21

84. Beukelaar LJ, Kroonenberg PM (1986). Changes over time in the relationship between hand preference and writing hand among left-handers. Neuropsychologia, 24(2): 301-3

85. Coren S, Halpern DF (1991). Left-handedness: a marker for decreased survival fitness. Psychol Bull, 109(1): 90-106

86. Persson PG, Allebeck P (1994). Do left-handers have increased mortality? Epidemiology, 5(3): 337-40

87. Martin WL, Freitas MB (2002). Mean mortality among Brazilian left- and right-handers: modification or selective elimination? Laterality, $7(1): 31-44$

88. Hicks RA, Johnson C, Cuevas T, Deharo D, Bautista J (1994). Do right-handers live longer? An updated assessment of baseball player data. Percept Mot Skills, 78(3 Pt 2): 1243-7

89. Bhushan B, Khan SM (2006). Laterality and accident proneness: a study of locomotive drivers. Laterality, 11(5): 395-404

90. Hicks RA, Inman G, Ching P, Bautista J, Deharo D, Hicks GJ (1998). Consistency of hand use and accidents with injury. Percept Mot Skills, 87(3 Pt 1): 851-4

91. Hicks RA, Pass K, Freeman H, Bautista J, Johnson C (1993). Handedness and accidents with injury. Percept Mot Skills, 77(3 Pt 2): 1119-22

92. Hicks RA, Inman G, Deharo D, Hicks GJ (1999). Consistency of hand use and frequent falls. Percept Mot Skills, 88(3 Pt 2): 1107-10

93. Porac C, Searleman A (2006). The relationship between hand preference consistency, health, and accidents in a sample of adults over the age of 65 years. Laterality, 11(5):
405-14

94. Hughes JR, Dorner E, Wind M (2008). Is the decreased longevity among left-handers related to an increase in heart disease? Clin EEG Neurosci, 39(4): 182-4

95. Ellis L, Engh T (2000). Handedness and Age of Death: New Evidence on a Puzzling Relationship. J Health Psychol, 5( 4): 561-565

96. Leask SJ, Beaton AA (2007). Handedness in Great Britain. Laterality, 12(6): 559-72

97. Iwasaki S (2000). Age and generation trends in handedness: an eastern perspective. Side bias: a neuropsychological perspective. In MK Mandal, MB Bulman-Fleming,G Tiwari. Dordrecht: Kluwer Academic Publishers.

98. Shimuzo A, Endo M (1983). Handedness and family sinistrality in a Japanese student population. Cortex, 19: 265272

99. Ellis SJ, Ellis PJ, Marshall E, Windridge C, Jones S (1998). Is forced dextrality an explanation for the fall in the prevalence of sinistrality with age? A study in northern England. J Epidemiol Community Health, 52(1): 41-4

100. Teixeira LA (2008). Categories of manual asymmetry and their variation with advancing age. Cortex, 44(6): 707-16

101. Rodrigues P, Lamboglia C, Cabral I, Barreiros J, Vasconcelos O (2009). Degree of hand preference in rightand left-handers: life-span age trends. Poster presented to International Seminar Challenges to Sport Sciences. Porto: FADEUP

102. Kalisch T, Wilimzig C, Kleibel N, Tegenthoff M, Dinse HR (2006). Age-related attenuation of dominant hand superiority. PLoS ONE, 1: e90

103. Herve PY, Leonard G, Perron M, Pike B, Pitiot A, Richer L, Veillette S, Pausova Z, Paus T (2009). Handedness, motor skills and maturation of the corticospinal tract in the adolescent brain. Hum Brain Mapp, 30(10): 3151-62

104. Beaton AA, Hugdahl K, Ray P (2000). Lateral asymmetries and interhemispheric transfer in aging: a review and some new data. Side bias: a neurophychological perspective. In MK Mandal, MB Bulman-Fleming,G Tiwari. Dordrecht: Kluwer Academic Publishers, 101-152

105. Francis KL, Spirduso WW (2000). Age differences in the expression of manual asymmetry. Experimental Aging Research, 26(2): 169-80

106. Goldstein G, Shelly C (1981). Does the right hemisphere age more rapidly than the left? J Clin Neuropsychol, 3(1): 65-78

107. Cabeza R (2001). Cognitive neuroscience of aging: contributions of functional neuroimaging. Scand J Psychol, 42(3): 277-86

108. Hausmann M, Gunturkun O, Corballis M (2003). Age-related changes in hemispheric asymmetry depend on sex. Laterality 8(3): 277-90

109. Dittmar M (2002). Functional and postural lateral preferences in humans: interrelations and life-span age differences. Hum Biol, 74(4): 569-85

110. De Agostini M, Khamis AH, Ahui AM, Dellatolas G (1997). Environmental influences in hand preference: an African point of view. Brain Cogn, 35(2): 151-67

111. Elalmis DD, Tan U (2008). Dynamics of manual skill: a computerized analysis of single peg movements and stochastic resonance hypothesis of cerebral laterality. Int $J$ Neurosci, 118(3): 399-432

112. Sommer IE, Aleman A, Somers M, Boks MP, Kahn RS 
(2008). Sex differences in handedness, asymmetry of the planum temporale and functional language lateralization. Brain Res, 1206: 76-88

113. Sato S, Demura S, Sugano N, Mikami H, Ohuchi T (2008). Characteristics of handedness in Japanese adults: influence of left-handed relatives and forced conversion. Int J Sport Health Sci, 6: 113-119

114. Annett M (1995). The Right Shift Theory of a Genetic Balanced Polymorphism for Cerebral-Dominance and Cognitive Processing. Cah Psychol Cognit Curr Psychol Cognit 14(5): 427-480.

115. McManus IC, Bryden MP (1992). The genetics of handedness, cerebral dominance, and lateralization. Handbook of neuropsychology. In I Rapin,SJ Segalowitz. Amsterdam: Elsevier Science Publishers, 115-144

116. McKeever WF (2000). A new family handedness sample with findings consistent with X-linked transmission. $\mathrm{Br} \mathrm{J}$ Psychol, 91 ( Pt 1): 21-39

117. Francks C, Maegawa S, Lauren J, Abrahams BS, VelayosBaeza A, Medland SE, Colella S, Groszer M, McAuley EZ, Caffrey TM, Timmusk T, Pruunsild P, Koppel I, Lind PA, Matsumoto-Itaba N, Nicod J, Xiong L, Joober R, Enard W, Krinsky B, Nanba E, Richardson AJ, Riley BP, Martin NG, Strittmatter SM, Moller HJ, Rujescu D, St Clair D, Muglia P, Roos JL, Fisher SE, Wade-Martins R, Rouleau GA, Stein JF, Karayiorgou M, Geschwind DH, Ragoussis J, Kendler KS, Airaksinen MS, Oshimura M, DeLisi LE, Monaco AP (2007). LRRTM1 on chromosome 2p12 is a maternally suppressed gene that is associated paternally with handedness and schizophrenia. Mol Psychiatry, 12(12): 1129-39, 1057

118. Geshwind N, Galaburda AM (1985). Cerebral lateralization: Biological mechanisms, associations and pathology: I. A hypothesis and a program for research. Arch Neurol, 42: 428-459

119. Mathews GA, Fane BA, Pasterski VL, Conway GS, Brook C, Hines M (2004). Androgenic influences on neural asymmetry: Handedness and language lateralization in individuals with congenital adrenal hyperplasia. Psychoneuroendocrinology, 29(6): 810-822

120. Nass R, Baker S, Speiser P, Virdis R, Balsamo A, Cacciari E, Loche A, Dumic M, New M (1987). Hormones and Handedness - Left-Hand Bias in Female Congenital Adrenal-Hyperplasia Patients. Neurology, 37(4): 711-715

121. Pfannkuche KA, Bouma A, Groothuis TGG (2009). Does testosterone affect lateralization of brain and behaviour? A meta- analysis in humans and other animal species. Philos Trans R Soc Lond B Biol Sci, 364(1519): 929-942

122. Nalcaci E, Kalaycioglu C, Cicek M, Genc Y (2001). The relationship between handedness and fine motor performance. Cortex, 37(4): 493-500

123. Lissek S, Hausmann M, Knossalla F, Peters S, Nicolas V, Gunturkun O, Tegenthoff M (2007). Sex differences in cortical and subcortical recruitment during simple and complex motor control: an fMRI study. Neuroimage, 37(3): 91226

124. Hausmann M, Kirk IJ, Corballis MC (2004). Influence of task complexity on manual asymmetries. Cortex, 40(1): 103-10

125. Bryden PJ, Roy EA (2005). A new method of administering the Grooved Pegboard Test: performance as a function of handedness and sex. Brain Cogn, 58(3): 258-68
126. Hoffstein V, Chan CK, Slutsky AS (1993). Handedness and sleep apnea. Chest, 103(6): 1860-2

127. Kaynar H, Dane S (2003). Prevalence of left-handedness among patients with different respiratory diseases. Int $J$ Neurosci, 113(10): 1371-7

128. Doody RS, Vacca JL, Massman PJ, Liao TY (1999). The influence of handedness on the clinical presentation and neuropsychology of Alzheimer disease. Arch Neurol, 56(9): 1133-7

129. Van Strien JW, Lagers-van Haselen GC, van Hagen JM, de Coo IFM, Frens MA, van der Geest JN (2005). Increased prevalences of left-handedness and left-eye sighting dominance in individuals with Williams-Beuren syndrome. $J$ Clin Exp Neuropsychol, 27: 967-976.

130. Suckling J, Roberts H, Walker M, Highley JR, Fenwick P, Oxbury J, Esiri MM (2000). Temporal lobe epilepsy with and without psychosis: exploration of hippocampal pathology including that in subpopulations of neurons defined by their content of immunoreactive calcium-binding proteins. Acta Neuropathol, 99(5): 547-54

131. Somers M, Sommer IE, Boks MP, Kahn RS (2009). Handpreference and population schizotypy: a meta-analysis. Schizophr Res, 108(1-3): 25-32 